\title{
Radar cross section study from wave scattering structures
}

\author{
S. Redadaa, A. Boualleg, N. Merabtine, and M. Benslama \\ Electromagnetism and Telecommunications Laboratory LET \\ Department of Electronics, Faculty of Engineering \\ University of Constantine, Algeria \\ E-mail: \{redasdz,bouadzdz\}@yahoo.fr; \{na_merabtine,malekbenslama\}@hotmail.com
}

\begin{abstract}
Radar remote sensing deals with the extraction of object information from electromagnetic wave parameters. To fully exploit the potential of acquiring quantitative information requires a detailed description of the microwaves scattering. The research on this topic was mostly centered on far-field analysis that assumes an incident plane wave, computation of its scattered field, and evaluation of the radar cross section. However, under certain practical conditions, the far-field analysis is not valid and a near-field analysis is necessary. In this paper, we have given a full analysis of the near-field of a wedge structure due to an incident wave field from a line source or a plane wave. The far-field pattern, for the case of a line source exciting the structure, is also analyzed.
\end{abstract}

Keywords: electromagnetic wave, scattering, structure, radar cross section.

Manuscript received 04.05.06; accepted for publication 23.10.06.

\section{Introduction}

The problem of electromagnetic wave scattering is very important in many applications, namely, remote sensing, antennas design and especially in defense applications. The research on this topic was mostly centered on far-field analysis that assumes an incident plane wave, computation of its scattered field due to the scatterer, and evaluation of the radar cross section (RCS) of the scatterer. When the transmitting and receiving antennas are far from the scatterer, the incident wave can be approximated by a plane wave and the scattered far-field can be regarded as the radiation far-field due to the induced currents on the scatterer, the far-field analysis thus applies. However, in practical applications, there are many situations, when the distance between the transmitting antenna and the scatterer is not large enough to treat the field arriving the scatterer as a plane wave, and the relative motion between the antennas and scatterer will produce the Doppler frequency shift. In these conditions, the far-field analysis is not valid and a near-field analysis is necessary [1].

In calculating the radar cross section of complex targets [2, 3], some parts of the structure can be modeled using singly curved sheets as, for example, the wings of an aircraft. For electrically large bodies, the geometrical theory of diffraction (GTD) [4,5] is a good high frequency technique to compute the scattering from those bodies. But, as is well known, that method is not valid in the caustic of reflected rays, which occurs, for example, when we illuminate a singly curved screen with a plane wave. Physical optics (PO) has been largely used in the last years to predict high frequency radar cross section problems, because, unlike geometrical optics and the GTD, it is valid in the transition regions and at caustics. PO can be improved using the fringe currents contribution of the edge currents of the physical theory of diffraction (PTD) [6, 7].

This paper is organized as follows. Section 2 provides the scattering analysis; we present the RCS formulae and possible approximations. We calculate field expressions for the problem of scattering by a twodimensional perfect electric conduction wedge capped with a dielectric cylinder. In Section 3, some numerical results for various configurations of the wedge structure are presented. Finally, a conclusion is given.

\section{Scattering analysis}

The RCS of a target characterizes its scattering property that is defined as the area intercepting the amount of power that, when scattered isotropically, produces in a receiver a density that is equal to the density scattered by the actual target. When the transmitter and receiver are in the same location, the RCS is referred to as monostatic (or backscattered), and it is referred to as bistatic when these two ones are located at different positions. 
For three-dimensional target, the RCS is given in terms of incident power density, magnetic field, and electric field [8]. The RCS in terms of electric field is given by

$$
\sigma_{3-D}=\lim _{\rho \rightarrow \infty}\left[4 \pi \rho^{2} \frac{\left|E^{s}\right|^{2}}{\left|E^{i}\right|^{2}}\right],
$$

where $\rho$ is the distance from the target to observation point, $E^{s}$ and $E^{i}$ are the scattered and incident electric field. Eq. (1) is valid when the target is illuminated by a plane wave that in practice can be only approximated when the target is placed in the far-field of the source, at least $\rho=2 D^{2} / \lambda$, where $D$ is the largest dimension of the target.

Consider a perfectly conducting rectangular thinflat plate in the $x-y$ plane as shown in Fig. 1. For a linearly polarized incident wave in the $x-y$ plane, the horizontal and vertical backscattered RCSs are, respectively, given by

$\sigma_{V}=\frac{b^{2}}{\pi}\left|\sigma_{1 V}-\sigma_{2 V}\left[\frac{1}{\cos \theta}+\frac{\sigma_{2 V}}{4}\left(\sigma_{3 V}+\sigma_{4 V}\right) \sigma_{5 V}^{-1}\right]\right|^{2}$,

$\sigma_{H}=\frac{b^{2}}{\pi}\left|\sigma_{1 H}-\sigma_{2 H}\left[\frac{1}{\cos \theta}-\frac{\sigma_{2 H}}{4}\left(\sigma_{3 H}+\sigma_{4 H}\right) \sigma_{5 H}^{-1}\right]\right|^{2}$,

where

$$
\begin{aligned}
& \sigma_{1 V}=\cos \left(k_{a} \sin \theta\right)-j \frac{\sin \left(k_{a} \sin \theta\right)}{\sin \theta}, \\
& \sigma_{2 V}=\frac{e^{j(k a-\pi / 4)}}{\sqrt{2 \pi}\left(k_{a}\right)^{3 / 2}}, \\
& \sigma_{3 V}=\frac{(1+\sin \theta) e^{-j k_{a} \sin \theta}}{(1-\sin \theta)^{2}}, \\
& \sigma_{4 V}=\frac{(1-\sin \theta) e^{j k_{a} \sin \theta}}{(1+\sin \theta)^{2}}, \\
& \sigma_{5 V}=1-\frac{e^{j\left(2 k_{a}-\pi / 2\right)}}{8 \pi\left(k_{a}\right)^{3}} ; \\
& \sigma_{1 H}=\left(\sigma_{1 V}\right)^{*}, \\
& \sigma_{2 H}=\frac{4 e^{j\left(k_{a}+\pi / 4\right)}}{\sqrt{2 \pi}\left(k_{a}\right)^{1 / 2}}, \\
& \sigma_{3 H}=\frac{e^{-j k_{a} \sin \theta}}{1-\sin \theta}, \quad \sigma_{4 H}=\frac{e^{-j k_{a} \sin \theta}}{1-\sin \theta}, \\
& \sigma_{5 H}=1-\frac{\left.e^{j\left(2 k_{a}+\pi / 2\right.}\right)}{2 \pi\left(k_{a}\right)} .
\end{aligned}
$$

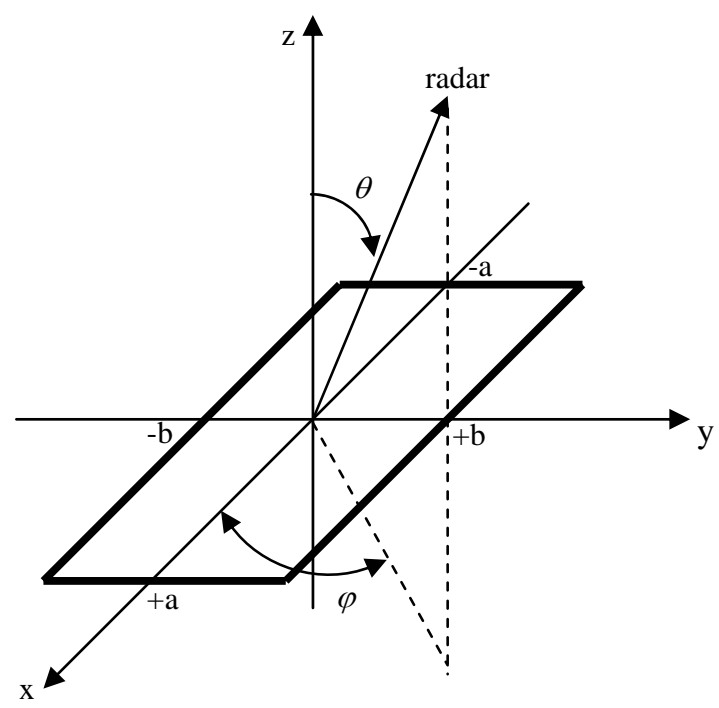

Fig. 1. Coordinates for the rectangular flat plate.

Here $k_{a}=k_{0} a, k_{0}$ is the free space wave number. Eqs (2) and (3) are valid quite accurate for the aspect angles $0 \leq \theta \leq 80^{\circ}$. For the aspect angles near $90^{\circ}$, Ross [9] obtained an empirical expression for the RCS by extensive fitting of measured data. It is given by

$\sigma_{H} \rightarrow 0$,

$$
\begin{aligned}
& \sigma_{V}=\frac{a b}{\lambda}\left\{\left[1+\frac{\pi}{2(2 a / \lambda)^{2}}\right]+\right. \\
& \left.+\left[1-\frac{\pi}{2(2 a / \lambda)^{2}}\right] \cos \left(2 k_{a}-\frac{3 \pi}{5}\right)\right\} .
\end{aligned}
$$

The backscattered RCS for a perfectly conducting thin rectangular plate for incident waves at any $\theta, \varphi$ can be approximated by

$$
\begin{aligned}
& \sigma=\frac{4 \pi a^{2} b^{2}}{\lambda^{2}}\left(\frac{\sin \left(a k_{0} \sin \theta \cos \varphi\right)}{a k_{0} \sin \theta \cos \varphi} \times\right. \\
& \left.\times \frac{\sin \left(b k_{0} \sin \theta \cos \varphi\right)}{b k_{0} \sin \theta \cos \varphi}\right)^{2}(\cos \theta)^{2} .
\end{aligned}
$$

Eq. (7) is independent of the polarization, and it is only valid for the aspect angles $\theta \leq 20^{\circ}$. Fig. 2 shows the backscattered RCS of a rectangular flat plate, for both vertical and horizontal polarizations.

The goal of analysis is to find the field expressions for the problem of scattering by a two-dimensional (2-D) perfect electric conduction (PEC) wedge capped with a dielectric cylinder as shown in Fig. 3. Using the cylindrical coordinates system, the excitation due to an electric line current of amplitude $I_{e}$ located at $\left(\rho_{0}, \varphi_{0}\right)$ result in transverse magnetic (TM) incident field with the electric field expression given by 

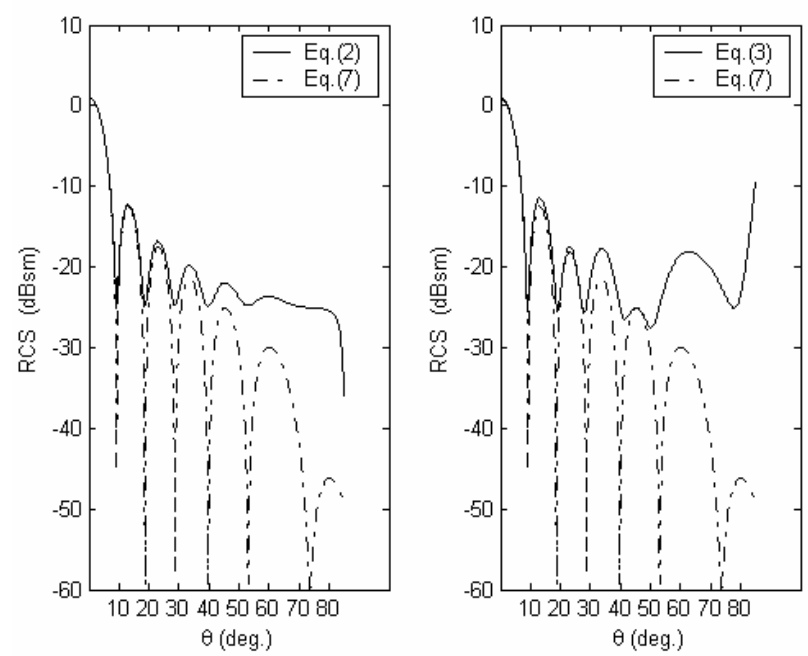

Fig. 2. Backscattered RCS for a rectangular flat plate of vertical (a) and horizontal (b) polarizations.

$$
E_{z}^{i}=-I_{e} \frac{\omega \mu_{0}}{4} H_{0}^{(2)}\left(k_{0}\left|\rho-\rho_{0}\right|\right)
$$

where $H_{0}^{(2)}$ is the Hankel function of the second kind of order zero.

The problem is divided into three regions I, II, and III. The field expressions may be assumed to take the following forms:

$E_{z}^{\mathrm{I}}=\sum_{n=0}^{\infty} a_{n} J_{v}\left(k_{1} \rho\right) \sin v(\varphi-\alpha) \sin v\left(\varphi_{0}-\alpha\right)$,

$E_{z}^{\mathrm{II}}=$

$=\sum_{n=0}^{\infty}\left(b_{n} J_{v}\left(k_{0} \rho\right)+c_{n} H_{v}^{(2)}\left(k_{0} \rho\right)\right) \sin v(\varphi-\alpha) \sin v\left(\varphi_{0}-\alpha\right)$,

$E_{Z}^{\mathrm{III}}=\sum_{n=0}^{\infty} d_{n} H_{v}^{(2)}\left(k_{0} \rho\right) \sin v(\varphi-\alpha) \sin v\left(\varphi_{0}-\alpha\right)$,

where $k_{1}$ is the wave number inside the dielectric,

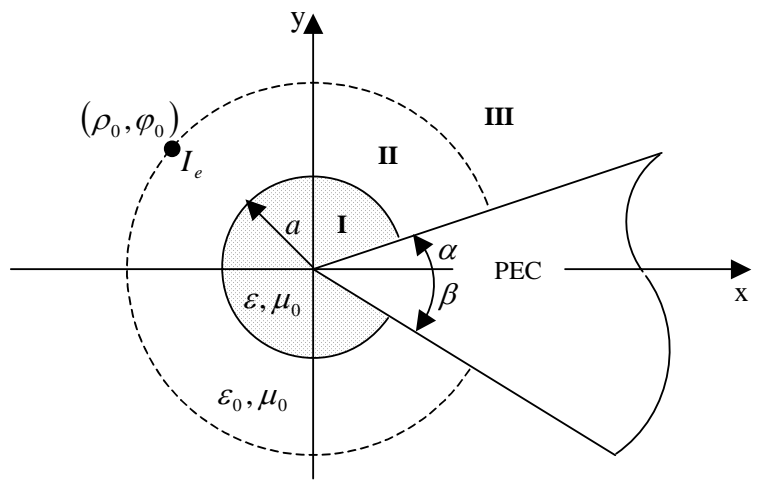

Fig. 3. Capped wedge structure.

$v=\frac{n \pi}{2 \pi-\alpha-\beta}$

while $J_{v}(x)$ and $H_{v}^{(2)}$ are the Bessel and Hankel functions of order $v$ and argument $x$. From the Maxwell equations, the magnetic field component $H_{\varphi}$ is related to the electric field component $E_{z}$ for a TM wave by

$$
H_{\varphi}=\frac{1}{j \omega \mu} \frac{\partial E_{Z}}{\partial \rho} \text {. }
$$

Thus, the magnetic field component $H_{\varphi}$ in the various regions may be written as $H_{\varphi}^{\mathrm{I}}=\frac{k_{1}}{j \omega \mu_{0}} \sum_{n=0}^{\infty} a_{n} J_{v}^{\prime}\left(k_{1} \rho\right) \sin v(\varphi-\alpha) \sin v\left(\varphi_{0}-\alpha\right)$,

$H_{\varphi}^{\mathrm{II}}=\frac{k_{0}}{j \omega \mu_{0}} \sum_{n=0}^{\infty}\left(b_{n} J_{v}^{\prime}\left(k_{0} \rho\right)+c_{n} H_{v}^{(2)^{\prime}}\left(k_{0} \rho\right)\right)$

$\sin v(\varphi-\alpha) \sin v\left(\varphi_{0}-\alpha\right)$,

$H_{\varphi}^{\mathrm{III}}=\frac{k_{0}}{j \omega \mu_{0}} \sum_{n=0}^{\infty} d_{n} H_{v}^{(2)^{\prime}}\left(k_{0} \rho\right) \sin v(\varphi-\alpha) \sin v\left(\varphi_{0}-\alpha\right)$,

where the prime indicates derivatives with respect to the full argument of the function. The boundary conditions require that the tangential electric field components vanish at the PEC surface. Also, the tangential field components should be continuous across the airdielectric surface and the virtual boundary between the regions I and II, except for the discontinuity of the magnetic field at the source point. Thus,

$E_{z}=0$ at $\varphi=\alpha, 2 \pi-\beta$,

$\left\{\begin{array}{c}E_{z}^{\mathrm{I}}=E_{z}^{\mathrm{II}} \\ H_{\varphi}^{\mathrm{I}}=H_{\varphi}^{\mathrm{II}}\end{array}\right.$ at $\rho=a$,

$\left\{\begin{array}{l}E_{z}^{\mathrm{II}}=E_{z}^{\mathrm{III}} \\ H_{\varphi}^{\mathrm{II}}=H_{\varphi}^{\mathrm{III}}\end{array}\right.$ at $\rho=\rho_{0}$.

The current density $J_{e}$ may be given in the Fourier series expansion as

$$
\begin{aligned}
& J_{e}=\frac{I_{e}}{\rho_{0}} \delta\left(\varphi-\varphi_{0}\right)=\frac{2}{2 \pi-\alpha-\beta} \frac{I_{e}}{\rho_{0}} \times \\
& \times \sum_{n=0}^{\infty} \sin v(\varphi-\alpha) \sin v\left(\varphi_{0}-\alpha\right) .
\end{aligned}
$$

The boundary condition on the PEC surface is automatically satisfied by the $\varphi$ dependence of the electric field Eq. (9). From the boundary conditions in Eq. (14) 
$\sum_{n=0}^{\infty} a_{n} J_{v}\left(k_{1 a}\right) \sin v(\varphi-\alpha) \sin v\left(\varphi_{0}-\alpha\right)=$

$=\sum_{n=0}^{\infty}\left(b_{n} J_{v}\left(k_{a}\right)+c_{n} H_{v}^{(2)}\left(k_{a}\right)\right) \sin v(\varphi-\alpha) \sin v\left(\varphi_{0}-\alpha\right)$,

$\frac{k_{1}}{j \omega \mu_{0}} \sum_{n=0}^{\infty} a_{n} J_{v}^{\prime}\left(k_{1 a}\right) \sin v(\varphi-\alpha) \sin v\left(\varphi_{0}-\alpha\right)=$

$\frac{k_{0}}{j \omega \mu_{0}} \sum_{n=0}^{\infty}\left(b_{n} J_{v}^{\prime}\left(k_{a}\right)+c_{n} H_{v}^{(2)^{\prime}}\left(k_{a}\right)\right) \sin v(\varphi-\alpha) \sin v\left(\varphi_{0}-\alpha\right)$.

From the boundary conditions in Eq. (15), we have

$\sum_{n=0}^{\infty}\left(b_{n} J_{v}\left(k_{0} \rho_{0}\right)+c_{n} H_{v}^{(2)}\left(k \rho_{0}\right)\right) \sin v(\varphi-\alpha) \sin v\left(\varphi_{0}-\alpha\right)$

$=\sum_{n=0}^{\infty} d_{n} H_{v}^{(2)}\left(k_{0} \rho_{0}\right) \sin v(\varphi-\alpha) \sin v\left(\varphi_{0}-\alpha\right)$,

$\frac{k}{j \omega \mu_{0}} \sum_{n=0}^{\infty}\left(b_{n} J_{v}^{\prime}\left(k_{0} \rho_{0}\right)+c_{n} H_{v}^{(2)^{\prime}}\left(k_{0} \rho_{0}\right)\right) \times$

$\times \sin v(\varphi-\alpha) \sin v\left(\varphi_{0}-\alpha\right)=$

$=\frac{k}{j \omega \mu_{0}} \sum_{n=0}^{\infty} d_{n} H_{v}^{(2)^{\prime}}\left(k_{0} \rho_{0}\right) \sin v(\varphi-\alpha) \sin v\left(\varphi_{0}-\alpha\right)-$

$-\frac{2}{2 \pi-\alpha-\beta} \frac{I_{e}}{\rho_{0}} \sum_{n=0}^{\infty} \sin v(\varphi-\alpha) \sin v\left(\varphi_{0}-\alpha\right)$.

Since Eqs (17) and (20) hold for all $\varphi$, the series of the left and right hand sides are equal term by term, more precisely,

$a_{n} J_{v}\left(k_{1 a}\right)=b_{n} J_{v}\left(k_{a}\right)+c_{n} H_{v}^{(2)}\left(k_{a}\right)$,

$k_{1} a_{n} J_{v}^{\prime}\left(k_{1 a}\right)=k\left(b_{n} J_{v}^{\prime}\left(k_{a}\right)+c_{n} H_{v}^{(2)^{\prime}}\left(k_{a}\right)\right)$,

$b_{n} J_{v}\left(k_{0} \rho_{0}\right)+c_{n} H_{v}^{(2)}\left(k_{0} \rho_{0}\right)=d_{n} H_{v}^{(2)}\left(k_{0} \rho_{0}\right)$,

$b_{n} J_{v}^{\prime}\left(k \rho_{0}\right)+c_{n} H_{v}^{(2)^{\prime}}\left(k \rho_{0}\right)=$

$=d_{n} H_{v}^{(2)^{\prime}}\left(k \rho_{0}\right)-\frac{2 j \eta_{0}}{2 \pi-\alpha-\beta} \frac{I_{e}}{\rho_{0}}$,

where $\eta_{0}$ is the characteristic impedance of free space.

From Eqs (21) and (23), we have

$a_{n}=\frac{1}{J_{v}\left(k_{1 a}\right)}\left[b_{n} J_{v}\left(k_{a}\right)+c_{n} H_{v}^{(2)}\left(k_{a}\right)\right]$,

$d_{n}=c_{n}+b_{n} \frac{J_{v}\left(k_{0} \rho_{0}\right)}{H_{v}^{(2)}\left(k_{0} \rho_{0}\right)}$.

After some mathematical operations, we get $b_{n}=-\frac{\pi \omega \mu_{0} I_{e}}{2 \pi-\alpha-\beta} H_{v}^{(2)}\left(k_{0} \rho_{0}\right)$.

Substituting $b_{n}$ in Eqs (21) and (22) and solving for $c_{n}$ yield

$$
\begin{aligned}
& c_{n}=\frac{\pi \omega \mu_{0} I_{e}}{2 \pi-\alpha-\beta} \times \\
& \times\left[H_{v}^{(2)}\left(k_{0} \rho_{0}\right) \frac{k J_{v}^{\prime}\left(k_{a}\right) J_{v}\left(k_{1 a}\right)-k_{1} J_{v}(k a) J_{v}^{\prime}\left(k_{1 a}\right)}{k_{0} H_{v}^{(2)^{\prime}}\left(k_{a}\right) J_{v}\left(k_{1 a}\right)-k_{1} H_{v}^{(2)}\left(k_{a}\right) J_{v}^{\prime}\left(k_{1 a}\right)}\right]
\end{aligned}
$$

From Eqs (26) through (28), $d_{n}$ may be given by

$d_{n}=\frac{\pi \omega \mu_{0} I_{e}}{2 \pi-\alpha-\beta} \times$

$\times\left[H_{v}^{(2)}\left(k_{0} \rho_{0}\right) \frac{k_{0} J_{v}^{\prime}\left(k_{a}\right) J_{v}\left(k_{1 a}\right)-k_{1} J_{v}\left(k_{a}\right) J_{v}^{\prime}\left(k_{1 a}\right)}{k H_{v}^{(2)^{\prime}}\left(k_{a}\right) J_{v}\left(k_{1 a}\right)-k_{1} H_{v}^{(2)}\left(k_{a}\right) J_{v}^{\prime}\left(k_{1 a}\right)}-\right.$

$\left.-J_{v}\left(k_{0} \rho_{0}\right)\right]$

with these closed form expressions for the expansion coefficients $a_{n}, b_{n}, c_{n}$ and $d_{n}$, the field components $E_{z}$ and $H_{\varphi}$ can be determined from Eqs (9) and (12), respectively. Alternatively, the magnetic field component $H_{\rho}$ can be computed from

$H_{\rho}=-\frac{1}{j \omega \mu} \frac{1}{\rho} \frac{\partial E_{Z}}{\partial \varphi}$.

Thus, the $H_{\rho}$ expressions for the three regions defined in Fig. 3 become

$H_{\rho}^{\mathrm{I}}=-\frac{1}{j \omega \mu \rho} \times$

$\times \sum_{n=0}^{\infty} a_{n} v J_{v}\left(k_{1} \rho\right) \cos v(\varphi-\alpha) \sin v\left(\varphi_{0}-\alpha\right)$,

$H_{\rho}^{\mathrm{II}}=-\frac{1}{j \omega \mu \rho} \times$

$\times \sum_{n=0}^{\infty} v\left(b_{n} J_{v}\left(k_{0} \rho\right)+c_{n} H_{v}^{(2)}\left(k_{0} \rho\right)\right) \cos v(\varphi-\alpha) \sin v\left(\varphi_{0}-\alpha\right)$,

$H_{\rho}^{\mathrm{III}}=-\frac{1}{j \omega \mu \rho} \times$

$\times \sum_{n=0}^{\infty} d_{n} v H_{v}^{(2)}\left(k_{0} \rho\right) \cos v(\varphi-\alpha) \sin v\left(\varphi_{0}-\alpha\right)$.

In the region III, the far scattered field may be found as the difference between the total and incident field. Thus, using Eqs (8) and (9) and considering the far-field condition $(\rho \rightarrow \infty)$ we get 

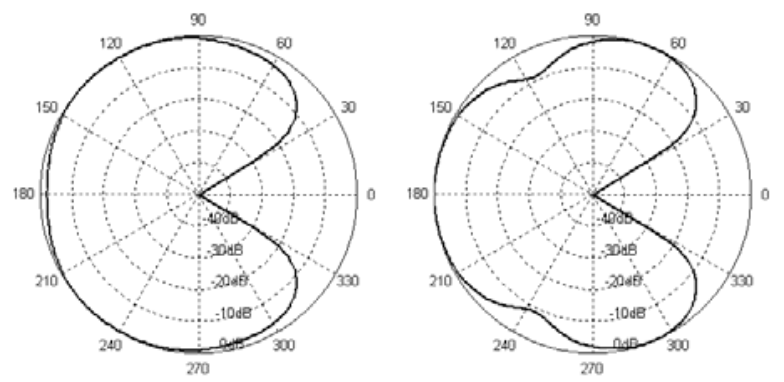

Fig. 4. Total far-field pattern of a line source near a conducting wedge with conducting-capped (a) and dielectriccapped edges (b).

$$
\begin{aligned}
& E_{z}^{\mathrm{III}}=E_{z}^{i}+E_{z}^{s}=\sqrt{\frac{2 j}{\pi k_{0} \rho}} e^{-j k \rho} \times \\
& \times \sum_{n=0}^{\infty} d_{n} j^{v} \sin v(\varphi-\alpha) \sin v\left(\varphi_{0}-\alpha\right) .
\end{aligned}
$$

For the plane wave excitation $\left(\rho_{0} \rightarrow \infty\right)$, the expressions in Eqs (27) and (28) reduce to

$$
\begin{aligned}
& b_{n}=-\frac{\pi \omega \mu_{0} I_{e}}{2 \pi-\alpha-\beta} j^{v} \sqrt{\frac{2 j}{\pi k_{0} \rho_{0}}} e^{-j k_{0} \rho_{0}}, \\
& c_{n}=\frac{\pi \omega \mu_{0} I_{e}}{2 \pi-\alpha-\beta} j^{v} \sqrt{\frac{2 j}{\pi k_{0} \rho_{0}}} e^{-j k_{0} \rho_{0}} \times \\
& \times \frac{k_{0} J_{v}^{\prime}\left(k_{a}\right) J_{v}\left(k_{1 a}\right)-k_{1} J_{v}\left(k_{a}\right) J_{v}^{\prime}\left(k_{1 a}\right)}{k_{0} H_{v}^{(2)^{\prime}}\left(k_{a}\right) J_{v}\left(k_{1 a}\right)-k_{1} H_{v}^{(2)}\left(k_{a}\right) J_{v}^{\prime}\left(k_{1 a}\right)},
\end{aligned}
$$

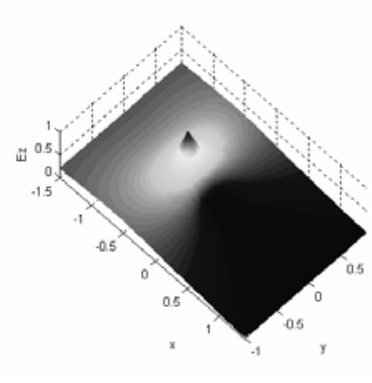

$a$
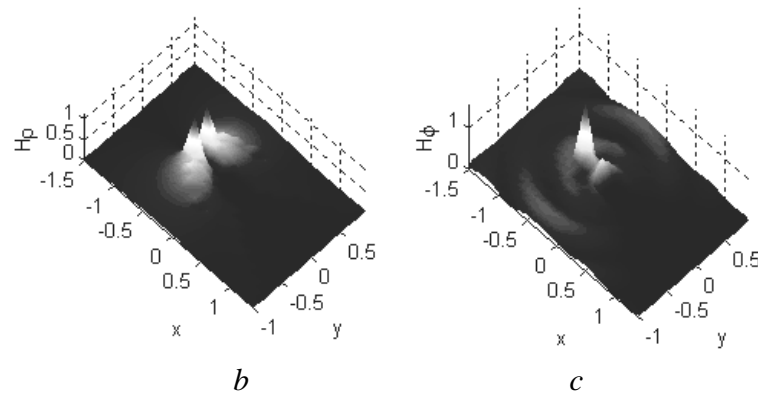

Fig. 5. Near-field patterns of a line source near a conducting wedge with a conducting-capped edge $E_{z}$ (a), $H_{\rho}$ (b), $H_{\varphi}$ (c). where the complex of the incident plane wave, $E_{0}$, can be given by

$E_{0}=-I_{e} \frac{\omega \mu_{0}}{4} \sqrt{\frac{2 j}{\pi k \rho_{0}}} e^{-j k_{0} \rho_{0}}$

in this case, the field components can be evaluated in the regions I and II only.

\section{Numerical results and discussion}

Fig. 2 presents the radar cross section of a rectangular flat plate for the vertical and horizontal polarizations, compared with the classical formulae. The parameters of structure are $a=b=10.16 \mathrm{~cm}$ and $f=300 \mathrm{MHz}$.

Fig. 4 presents the far-field of a capped wedge in the presence of an electric line source field. We clearly show how the cap parameters affect the maximum radiation of the line source in the presence of wedge. The distribution of the components of the fields on the near-field of two cases (conducting capped edge, dielectric capped edge) is computed and shown in Figs 5 and 6. The near-field distribution for an incident wave field of these two types of wedges is also computed and shown in Figs 7 and 8. These near-field distributions clearly demonstrated the effect of cap parameters in altering the sharp edge singular behaviour. We have used the following wedge structure parameters: $a=0.15 \mathrm{~cm}, \rho_{0}=0.5 \mathrm{~cm}, \alpha=\beta=30^{\circ}, \varepsilon_{\gamma}=3, I_{e}=1 \mathrm{~mA}$.
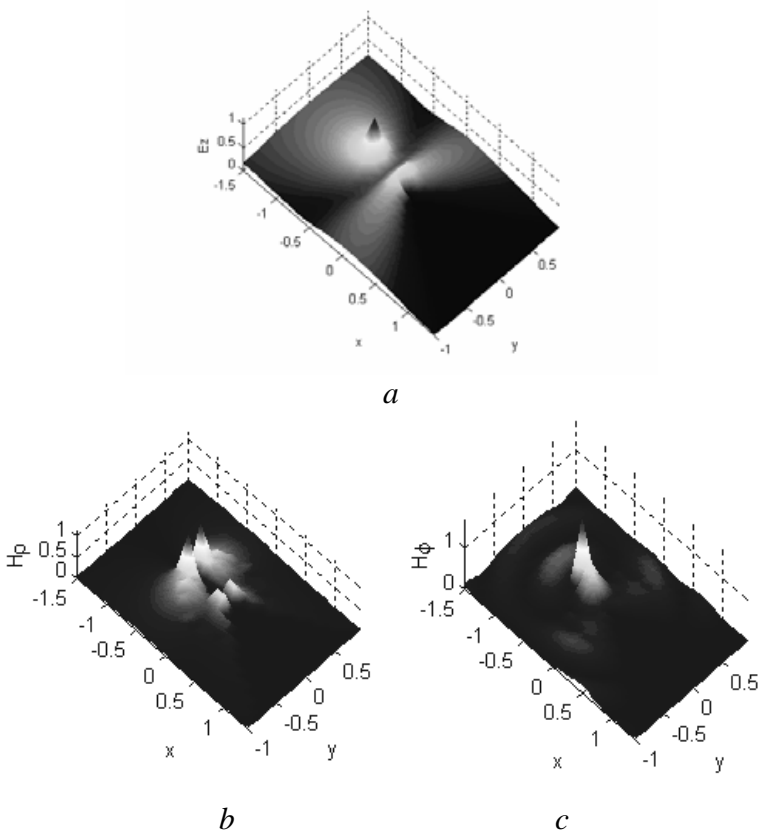

Fig. 6. Near-field patterns of a line source near a conducting wedge with a dielectric-capped edge $E_{z}(\mathrm{a}), H_{\rho}$ (b), $H_{\varphi}$ (c). 


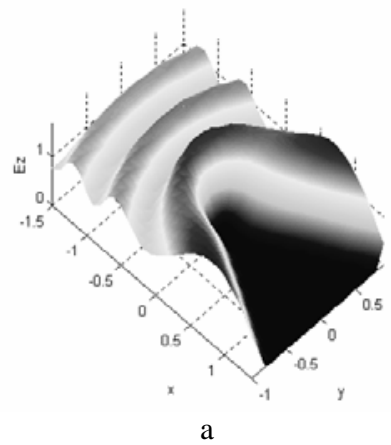

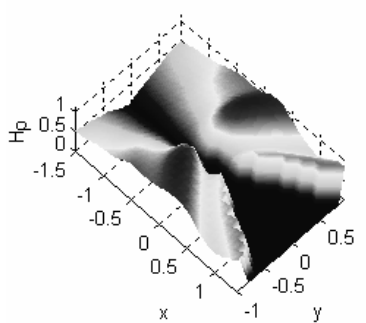

b

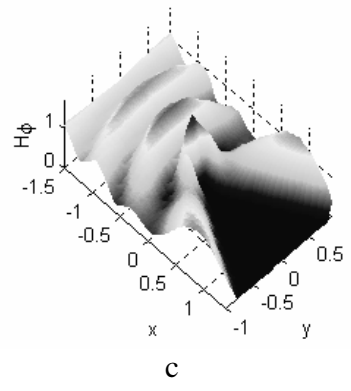

Fig. 7. Near-field patterns of the plane wave incident on a conducting wedge with a conducting-capped edge $E_{z}$ (a), $H_{\rho}$ (b), $H_{\varphi}$ (c).

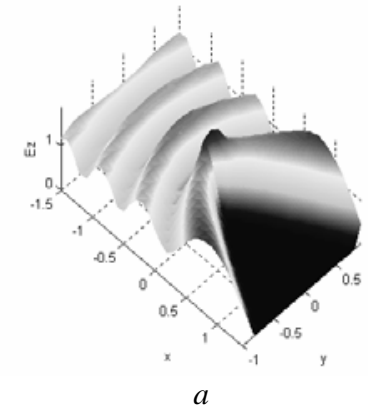

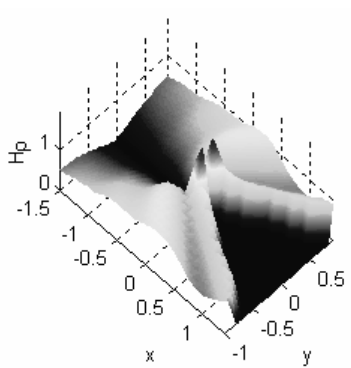

b

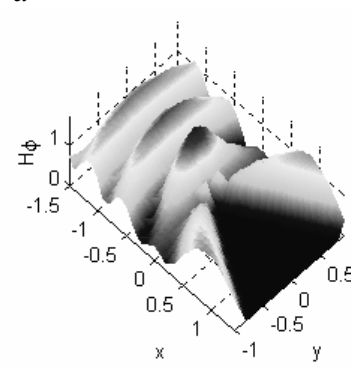

$c$
Fig. 8. Near-field patterns of the plane wave incident on a conducting wedge with a dielectric-capped edge $E_{z}$ (a), $H_{\rho}$ (b), $H_{\varphi}$ (c).

\section{Conclusion}

In this paper, we have presented a full analysis of electromagnetic scattering. We have presented a case of backscattered radar cross section for a rectangular flat plate. The analysis of the far- and near-field patterns for a wedge structure shows the effect of cap parameters on the maximum radiation of the line source. We have also examined the effect the cap parameters on the sharp edge behavior for an incident plane wave.

\section{References}

1. S.K. Jeng, Near-field scattering by physical theory of diffraction and shooting and bouncing rays // IEEE Trans. Ant. Propag. 46(4), p. 551-558 (1998).

2. N.N. Youssef, Radar cross section of complex targets // Proc. IEEE 77(5), p. $722-734$ (1989).

3. D. Colton and R. Kress, Integral equation methods in scattering theory. Wiley-Interscience, 1983.

4. R.G. Kouyoumjian and P.H. Pathak, A uniform geometrical theory of diffraction for an edge in a perfectly conducting surface // Proc. IEEE, 62(11), p. 1448-1461 (1974).

5. S.M. Rao, D.R. Wilton, and A.W. Glisson, Electromagnetic scattering by surfaces of arbitrary shape // IEEE Trans. Ant. Propag. AP-30(3), p. 409-418 (1982).

6. E.F. Knott, J.F. Shaeffer and M.T Tuley, Radar cross section: Its prediction, measurement and reduction. Artech House, Dedham, MA, 1985.

7. S. Blume and V. Krebs, Numerical evaluation of dyadic diffraction coefficients and bistatic radar cross sections for a perfectly conducting semiinfinite elliptic cone // IEEE Trans. Ant. Propag. 46(3), p. 414- 424 (1998).

8. C.A. Balanis, Antenna theory: Analysis and design. John Wiley \& Sons, 2nd edition, New York, 1997.

9. R.A Ross, Radar cross section of rectangular flat plate as function of aspect angle // IEEE Trans. Ant. Propag. AP-14, p. 329-335 (1966). 